\title{
Intergalactic HI Clouds
}

\author{
F.H. Briggs \\ RSAA, Mount Stromlo Observatory, ANU \\ Cotter Road, Weston Creek 2611 ACT, Australia
}

\begin{abstract}
Neutral intergalactic clouds are so greatly out numbered by galaxies that their integral HI content is negligible in comparison to that contained in optically luminous galaxies. In fact, no HI cloud that is not associated with a galaxy or grouping of galaxies has yet been identified. This points to a causal relationship that relies on gravitational potentials that bind galaxies also being responsible for confining HI clouds to sufficient density that they can become self-shielding to the ionizing background radiation. Unconfined clouds of low density become ionized, but confined clouds find themselves vulnerable to instability and collapse, leading to star formation.
\end{abstract}

\section{Introduction}

Astronomers think of hydrogen as the most primitive of the atomic elements. Hydrogen, helium, and a trace of lithium, along with their isotopes, constitute the primordial mix of baryonic material emanating from The Big Bang origin of the Universe; the heavier elements form over time through nuclear processes in the interiors of successive generations of stars.

A natural consequence has been the quest to identify remnant clouds of primordial hydrogen in a pristine state. The suspicion a few decades ago was that clouds of neutral atomic hydrogen might float freely in the intergalactic medium, where they would exist as self-gravitationally bound systems in much the same way that galaxies of stars are bound together. Subsequently, astronomers have succeeded in identifying the ionized intergalactic medium through the discovery of the Lyman- $\alpha$ forest, but they have yet to find free-floating clouds of neutral gas.

This contribution to these proceedings gives a short review of the limits on intergalactic neutral clouds, along with a discussion of why we no longer expect them to exist. It is important to point out from the start that there are numerous HI clouds swarming around galaxies and groups. In one sense, one could argue that these clouds "between the galaxies" might qualify as intergalactic. However, in all well-studied cases, these clouds appear to be products of their environments (possible tidal or interaction remnants), and their existence in the neutral phase seems to be a consequence of confinement by the gravitational potentials of the host galaxies or groups. HI clouds are not found unaccompanied by galaxies. 


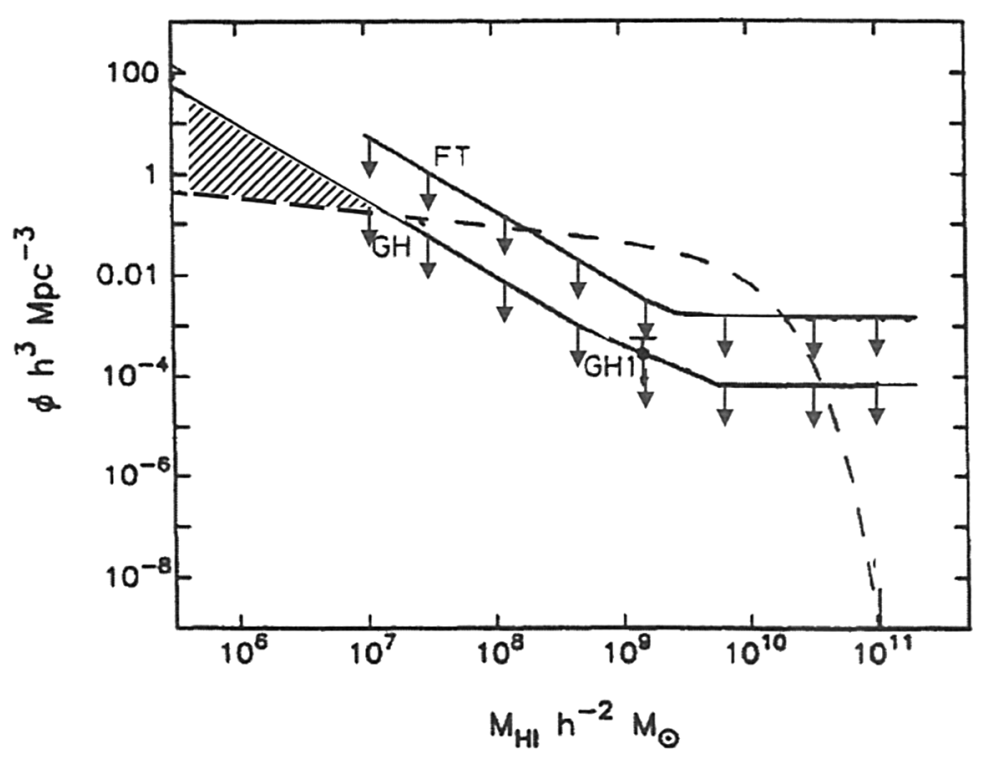

Figure 1. The HI mass function $\phi\left(M_{H I}\right)$ compared with upper limits to the number densities of intergalactic HI clouds (after Briggs 1990) per $\mathrm{Mpc}^{3}$ per decade of $\mathrm{HI}$ mass. The upper limits derived by Fisher \& Tully (1981) (upper solid curve with arrows) and Briggs (1990) (lower solid curve with arrows) are compared to the HI mass function for galaxies (dashed curve) as derived by Briggs (1990) using the optical luminosity function of Felton (1985) and the HI to optical luminosity ratio trend from Fisher \& Tully (1975). The hatched region at left is the only zone where the number of intergalactic clouds might have exceeded the number of galaxies of the same HI mass.

\section{Limits to the numbers of intergalactic $\mathrm{HI}$ clouds}

A number of low redshift $21 \mathrm{~cm}$ line surveys for intergalactic $\mathrm{HI}$ clouds took place in the 1970's, and the resulting trend of associating all HI signals with optically luminous galaxies has continued to this day. Fisher and Tully (1981) summarized the surveys at that time, and they concluded that the mass in intergalactic clouds in the mass range $10^{7}$ to $10^{10} M_{\odot}$ is less than $6 \%$ of the luminous mass in galaxies and less than $5 \times 10^{-4}$ the density required for a closed Universe. With $98 \%$ of their survey of 1787 nearby galaxies completed, they had found no convincing detection of an HI signal without an optical counterpart.

There is a clear trend of increasing HI-mass to optical luminosity ratio $M_{H I} / L$ with decreasing optical luminosity for late-type spiral and irregular galaxies (Fisher \& Tully 1975). The early survey by Lo \& Sargent (1979) discovered several dwarf galaxies in nearby groups, and some of these had $M_{H I} / L>10$. One might argue that these could be called "HI clouds with a trace of stellar 


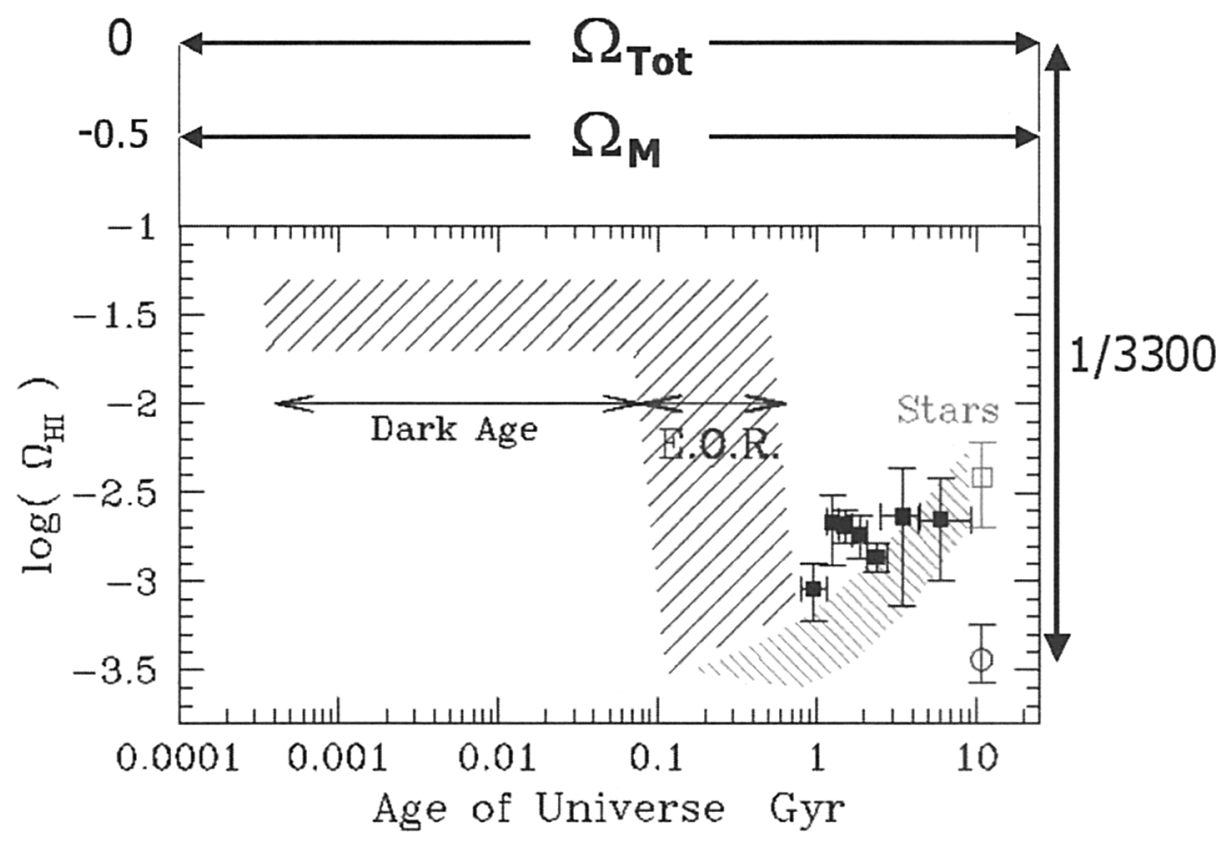

Figure 2. History of the neutral hydrogen content of the Universe. The logarithm of the neutral gas density normalized to the 'closure density' necessary to close the Universe is plotted as a function of the age of the Universe. Square filled points are measurements from Damped Lyman- $\alpha$ QSO absorption-line statistics. The open circle at far right represents the neutral gas content of the present day $(z=0)$ Universe. For comparison, the rising trend of stellar mass content appears has a hatched envelope, which increases to the value measured at $z=0$ from the optical luminosity density of stars.

contamination," although in these cases, the optical observations indicate dwarf galaxies of ordinary appearance... they are simply extremely rich in HI (Warren, Jerjen et al, this symposium).

Briggs (1990) revisited the upper limits to intergalactic clouds and compared them to the number density of HI-rich galaxies by deriving $\phi\left(M_{H I}\right)$ the HI mass function (HIMF) from the properties of the optically identified galaxy populations. Felton's (1985) optical luminosity function (with faint-end power law exponent of $\alpha=-1.25$ and the $M_{H I} / L(L)$ trend from Fisher and Tully (1975) led to the HIMF shown in Fig. 1. In the mass range around $M_{H I} \sim 10^{9} M_{\odot}$, the Fisher-Tully limits constrain the intergalactic HI clouds to be less numerous than galaxies by a factor of ten. In addition, Briggs reviewed the $21 \mathrm{~cm}$ galaxy surveys made in the decade since Fisher and Tullys' catalog. The important Arecibo surveys of Giovanelli and Haynes and colleagues (1989a) had turned up the "Virgo Cloud" (Giovanelli \& Haynes 1989b), a Magellanic irregular galaxy that had been mistaken for an intergalactic cloud, and the confidence of the detection of this object with $M_{H I} \sim 10^{9} M_{\odot}$ in an Arecibo calibration scan rein- 
forced the idea that the Arecibo surveys could place strict constraints on the cloud population. These types of surveys, which are designed to study optically selected galaxies, rely on a calibration scan from blank sky to go with every observation with the telescope aimed at a galaxy. The number of objects discovered by chance in the off-source scans provides a measure of the intergalactic cloud detection rate and places upper limits on their space density. The upper limits then become quite strict over the mass range $10^{8}$ to $10^{10.2} M_{\odot}$, constraining the population to be a factor of 10 to 100 less numerous than galaxies of similar mass over this range.

Only in the mass regime $M_{H I}<10^{7} M_{\odot}$ do the upper limits permit HI clouds to outnumber galaxies. The upper limits to space density still constrain the integral mass density of such low mass clouds to be negligible in comparison to the mass content of the massive, luminous galaxies that are the dominant reservoirs of atomic hydrogen at the present age of the Universe.

The integral $\mathrm{HI}$ content of the Universe at present is computed by the integral $\rho_{H I}=\int M_{M I} \phi\left(M_{H I}\right) d M_{H I}$. The HIMF from Briggs (1990) yields a value for $\rho_{H I}$ of $7 \times 10^{7} M_{H I} \mathrm{Mpc}^{-3}$ with a faint-end HIMF slope of $\alpha=-1.28$ $\left(H_{o}=75 \mathrm{~km} \mathrm{~s}^{-1} \mathrm{Mpc}^{-1}\right)$. Recent values from the Parkes HIPASS survey (Zwaan et al 2003) are $\rho_{H I}=6.1 \times 10^{7} M_{H I} \mathrm{Mpc}^{-3}$ with $\alpha=-1.3$, indicating a convergence between measurements based on optical and radio selection techniques.

A comprehensive understanding of the Galactic High Velocity Cloud population remains elusive. Recently, Blitz et al (1999) and Braun and Burton (1999) have explored the possibility that the in-falling HVC population of $\mathrm{HI}$ clouds are remnants of a primordial extragalactic population of dark matter mini-halos. In this scenario, the HI masses of the clouds would typically be larger than $\sim 10^{7} M_{\odot}$, and every large galaxy should be surrounded by a similar halo of a few hundred of these objects, assuming the phenomenon is a genuine and common feature of galaxy formation and evolution. The fact that nearby galaxies and groups do not possess such a halo of small clouds (Zwaan \& Briggs 2000, Zwaan 2001) has ruled out this idea, requiring that the clouds must be at least an order of magnitude less massive and fall at distances within $\sim 200 \mathrm{kpc}$ of the Milky Way, well within our Galaxy's halo.

The clear association of neutral gas clouds with star-bearing galaxies implies that the HI relies on the confinement of the galaxies' gravitational potentials for their survival.

\section{What happened to the HI clouds that formed at Recombination?}

Figure 2 gives an overview of the history of neutral gas clouds over the age of the Universe. It begins at the phase transition corresponding to the release of the Cosmic Microwave Background (at $z \sim 1100$ ), when the ionized baryons and electrons combine to become a neutral gas that is transparent to the CMB photons. There follows the only period, lasting about 100 million years, when the majority of the Universe's atoms are neutral. This period, known as the 'Dark Age,' ends when the first objects collapse as a result of gravitational instability, providing sources of ionizing energy. We refer to the end of the Dark Age as the 'Epoch of Reionization' (EoR), and we associate the EoR with the onset of the first generation of stars (which form in the most over-dense regions) and 
the appearance of protogalactic objects, which become the building blocks of galaxies.

In the diagram of Fig. 2, the EoR is also marked by the appearance of a second shaded region that indicates schematically the beginnings of the build up of mass in stars, as subsequent generations of star formation gradually lock increasing numbers of baryons into low mass, long lived stars. The stellar mass content of the Universe rises steadily from the EoR to the present, where we have precise measurements through meticulous inventories of the numbers of galaxies and their luminosities (i.e., the galaxy luminosity function and the integral luminosity density)(see for instance, Madgwick et al 2002). The neutral hydrogen content at $z \approx 0$ is measured by $21 \mathrm{~cm}$ line surveys (Zwaan et al 1997 , Zwaan et al 2003) such as those described in the previous section.

Through the period following the EoR, astronomers have statistical measures of the HI content as a function of time through the observation of QSO absorption lines. Any gas rich object that populates the Universe has a random chance of intervening along the line sight to distant objects. Quasi-stellar objects are especially useful as background sources since they have strong optical and UV continuum emission against which intervening gas clouds can imprint a distinctive absorption line spectrum. In the case of thick clouds of neutral gas, the Lyman- $\alpha$ line of $\mathrm{HI}$ is so strong that it presents an easily recognized 'damping wing' profile, which has led to the Damped Lyman- $\alpha$ (DLA) class of QSO absorption line (Wolfe et al 1986); in the minds of most astronomers, the DLAs are associated with gas-rich protogalaxies, which are the precursors of the larger galaxies that we observe around us at present (Prochaska \& Wolfe 1997, Haenelt et al 1998).

\subsection{The ionization/recombination competition}

Once sources of ionization formed in the earliest astrophysical structures, the survival of neutral clouds became a competition between ionization and recombination rates. Since ionization is such a common hazard to the existence of neutral atoms, it is natural to ask, "how rapidly can an ion recover through recombination, after it has chanced to become ionized?" For hydrogen, the recombination rate $R$ is easily computed (for instance Spitzer 1978), and the time $t_{\text {recomb }}$ it takes for recombination to eliminate the electrons in a cloud of electron density $n_{e}$ is

$$
t_{\text {recomb }}=\frac{n_{e}}{R}=\frac{n_{e}}{n_{e} n_{p} \alpha_{\text {recomb }}}=\frac{1}{n_{p} \alpha_{\text {recomb }}} \approx \frac{T^{1 / 2}}{2 \times 10^{-11} n_{p}} \mathrm{sec}
$$

where $n_{p}$ is the proton density and $\alpha_{\text {recomb }}$ is the recombination coefficient. To get a feeling for the vulnerability of the bulk of the baryons that populate the intergalactic medium, the number density of baryons $n_{\text {baryon }}$ forms an estimate of $n_{p}$; over-dense regions will have relatively shorter recombination times. In an expanding Universe, $n_{p} \sim n_{\text {baryon }} \sim(1+z)^{3}$, so that

$$
t_{\text {recomb }} \propto \frac{T^{1 / 2}}{(1+z)^{3}}
$$

The recombination time of the IGM at mean density has a strong dependence on age of the Universe through the $(1+z)^{3}$, and a modest dependence on 


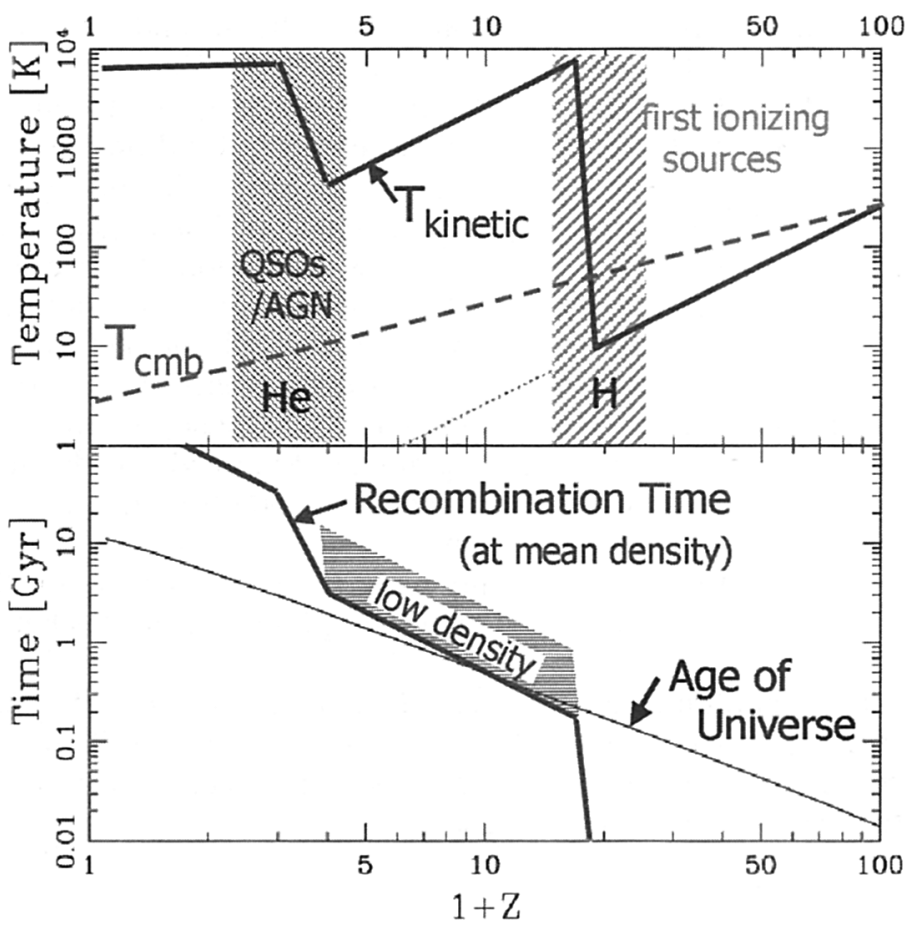

Figure 3. Recombination time in the intergalactic medium as a function of redshift $z$. Upper Panel: Kinetic temperature $T_{k}$ and CMB temperature $T_{C M B}$ vs. redshift. Episodes of heating through photoionization of hydrogen occur during the Epoch of Reionization and during the reionization of helium at a later time by the harder radiation from active galactic nuclei. Lower Panel: Recombination Time for an intergalactic medium of mean baryonic density, compared with the Age of the Universe as a function of redshift.

temperature $T$. Fig. 3 provides a rough illustration of how the IGM temperature varies with time and the net influence of the dependencies in Eqn. 2 on the ionization state of the Universe.

If the expansion of the Universe would allow a completely uniform expansion of the IGM without the growth of gravitationally-driven density instabilities, the gas kinetic temperature would decline in the adiabatic expansion with dependence $T_{k} \propto(1+z)^{2}$. At the same time, the CMB radiation temperature declines as $T_{C M B} \propto(1+z)$, causing the two temperatures to decouple after $z \approx 100$, when electron scattering ceases to be effective. The IGM is reheated when photoionization spreads through the medium generating energetic photoelectrons that deposit their kinetic energy through scattering. Once the IGM is fully ionized, there is no effective means of adding energy to the gas, since the photons generated by the stars can now flow uninhibited through a transparent medium, and the IGM again cools adiabatically due to Universal expansion. 
A similar heating event can occur during the age around $z \sim 2$ when QSOs are most common. QSOs, as well as lesser AGN, radiate photons that are capable of ionizing helium, and these harder photons generate photoelectrons throughout the IGM, providing a second round of localized heating.

The two heating events impact on the ability of the Universe to recombine. The lower panel of Fig. 3 compares the recombination time $t_{\text {recomb }}$ of an IGM of mean density to the age of the Universe $t_{\text {age }}$ as a function of redshift. If $t_{\text {recomb }}$ is long compared to $t_{\text {age }}$, the IGM would never recover from its ionized state, even if the source of ionizing photons were turned off completely. The figure shows that there is period between the two heating events, when recombination can compete with ionization, depending on 1) the intensity of the ionizing flux and 2) the local density. Under-dense regions would already be destined to stay forever ionized. Over-densities, especially those clouds confined in gravitational potential wells, may be able to recombine.

At low redshifts, the density of the mean IGM has become so dilute, that the IGM will remain ionized, even though the photoionizing background from AGN tails off.

The existence of atomic hydrogen clouds at all at low redshift in and around galaxies is due to their confinement to high density (greater than $\sim 0.1 \mathrm{~cm}^{-3}$ ) where the recombination times are $<10^{5} \mathrm{yrs}$, and recombination can compete effectively to make self-shielding clouds.

\section{Summary}

There is no evidence that truly intergalactic neutral hydrogen clouds exist at the present age of the Universe. The observational evidence constrains them to constitute a small fraction of the neutral gas contained in galaxies and to be at most a tiny fraction of the Universal baryons. The bulk of the baryons appear to be contained in the ionized intergalactic medium and stars. Where the hydrogen is neutral, it is confined in galactic potential wells, where 1) it is self-shielding against ionizing radiation by having sufficient density that recombination can compete with the rate ionization and 2) it is vulnerable to the instabilities that lead to star formation.

There is a limited range of options for neutral gas in a dark matter halo. The confining potential of a low mass halo (with virial temperature below $\sim 10^{4} \mathrm{~K}$ ) that has formed early as part of the first star forming protogalaxies will be unable to retain its gas during the first bursts of star formation, and the halo will be evacuated. Low mass halos that form later (after reionization and reheating), will be unable to capture and confine hot baryons from the intergalactic medium, and they therefore are destined to remain free of neutral gas. Massive halos can retain and capture gas, but, once confined in a halo, the gas is susceptible to collapse and star formation. The strong association of $\mathrm{HI}$ with star bearing halos makes sense.

In fact, there is a further consequence of this apparently complete correlation: Since all neutral gas appears to be associated in some way with stellar light, this constitutes evidence that there are no dark Dark Matter halos that are comparably massive to the Dark Matter halos that host galaxies, since such halos would be able to compress gas clouds in much the same way that the outer disks 
and rings of material hover in the outer regions of late-type galaxies (Schneider et al 1989, van Driel \& van Woerden 1991, Oosterloo et al 2003). Since rings or isolated clouds without stars are not observed, only a small minority of halos of galaxy-sized mass that are devoid of stellar emission can exist.

Acknowledgments. The author is grateful to the organizers of the Symposium for assembling a fascinating meeting and to the editors of the proceedings for their patience.

\section{References}

Blitz, L. et al 1999, ApJ, 514, 818

Braun, R. and Burton, W.B. 1999, A\&A, 341, 437

Briggs, F.H. 1990, AJ, 100, 999

van Driel, W., \& van Woerden, H. 1991, A\&A, 243, 71

Felton, J.E. 1985, Comments Astrophys., 11, 53

Fisher, J.R., \& Tully, R.B. 1975, A\&A, 44, 151

Fisher, J.R., \& Tully, R.B. 1981, ApJL, 243, L21

Giovanelli, R., \& Haynes, M.P. 1989a, AJ, 97, 633

Giovanelli, R., \& Haynes, M.P. 1989b, ApJL, 346, L5

Haehnelt, M., Steinmetz, M., Rauch, M. 1998, ApJ, 495, 647

Lo, K-Y., \& Sargent, W.L.W. 1979,

Madgwick, D.S. et al 2002, MNRAS, 333, 133

Oosterloo, T. et al. 2003, IAUS, 217, 108

Prochaska, J.X., Wolfe, A.M. 1997, ApJ, 487, 73

Schneider, S.E., et al 1989, AJ, 97, 666

Spitzer, L. 1978, Physical Processes in the Interstellar Medium, John Wiley \& Sons: New York.)

Wolfe, A.M., et al 1986, ApJS, 61, 249

Zwaan, M.A., et al 1997, ApJ, 490, 173

Zwaan, M.A., \& Briggs, F.H. 2000, ApJ, 530L, 61

Zwaan, M.A. 2001, MNRAS, 325, 1142

Zwaan, M.A., et al, 2003, AJ, 125, 2842 